\title{
Quantitative Phase Analysis of Rapid Solidification Products in Al-Cu Alloys by Automated Crystal Orientation Mapping in the TEM.
}

\author{
K. W. Zweiacker ${ }^{1}$, M. A. Gordillo ${ }^{1}$, C. Liu ${ }^{1}$, J. T. McKeown ${ }^{2}$, G. H. Campbell ${ }^{2}$, T. LaGrange ${ }^{3}$, B. W. \\ Reed $^{3}$, J. M. Wiezorek ${ }^{1}$ \\ 1. University of Pittsburgh, Pittsburgh, PA, USA \\ 2. Lawrence Livermore National Laboratory, Livermore, CA, USA \\ 3. Integrated Dynamic Electron Solution, Inc, Pleasanton, CA, USA
}

There has been significant interest in laser-based-processing methods for the manufacturing of complex components (e.g. additive manufacturing) or post-processing/repair work (e.g. laser welding). Since laser processing tends to result in the formation of rapidly solidified microstructures, it becomes increasingly important to understand the microstructural development under such non-equilibrium conditions. Pulsed-laser-based-processing methods have been foci of investigations on rapidly solidified microstructures. This method has been shown to produce unique microstructures and micro-constituents in metallic thin films at the nanometer-scale [1].

Recently, in-situ dynamical transmission electron microscopy (DTEM) studies on the rapid solidification of hypo-eutectic Al-Cu alloys have identified a modulation of the microstructural features as a function of solid-liquid interface motion [2]. Elucidating crystallographic dependencies during rapidly solidifying crystal growth in multi-phase alloys systems is of fundamental importance in understanding laser-assisted solidification processes [3-5]. However, due to the nm-scale of the resulting microstructural features, acquiring data sets with the appropriate spatial-resolution is challenging with electron backscatter diffraction (EBSD) based orientation image mapping (OIM) in scanning electron microscopes. A promising alternative analytical technique, which offers nm-spatial resolution, is precession electron diffraction (PED) based automated crystal orientation mapping (ACOM) in the TEM. This technique provides statistically significant and representative data sets for mapping of crystal orientations and spatial distribution of crystalline phases for instance.

Here we report an investigation of the microstructural development in a pulsed-laser melted rapidly solidified thin film alloy of Al-3at. \% Cu. Under equilibrium conditions this hypoeutectic Al-alloy contains the face-centered cubic $\alpha$-Al matrix and tetragonal $\theta-\mathrm{Al}_{2} \mathrm{Cu}$ precipitate phases. Solid-liquid interface velocities obtained via in-situ DTEM experiments as high as $1.5 \mathrm{~m} / \mathrm{s}$ are reached during solidification. These high interface velocities produce microstructures that deviate from those expected under more conventional conditions. Figure 1a is a collection of bright field TEM images of a representative melt-pool produced from laser irradiation exhibiting the different morphological zones arising during rapid solidification, e.g. Heat-affected zone, Columnar grain growth, Banded microstructure. Figure $1 \mathrm{~b}$ is an expanded view of the melt-pool edge region, which shows the columnar growth zone with fine-scale copper rich precipitates, identified as the metastable $\mathrm{Al}_{2} \mathrm{Cu}\left(\theta^{\prime}\right)$ phase. The crystallographic orientation relationships (OR) of Al matrix and precipitates were determined by ACOM (e.g. Figure 2). The Al grains are aligned such that the $\langle 100\rangle$ direction is parallel to the crystal growth , i.e. solidification, direction. A large proportion of the precipitates adopt an OR of $\{001\}_{\theta}, \|\{110\}_{\alpha} \&$ $<110>_{\theta}, \|<100>_{\alpha}$ (Fig. 2b), and the minority adopt the expected 'cube' OR of $\{001\}_{\theta}, \|\{100\}_{\alpha} \&$ $\left.<100\rangle_{\theta}, \|<100\right\rangle_{\alpha}$ (Fig. 2c). However, many precipitates do not exhibit any low-index OR with the Al matrix. The statistically significant analysis shows clearly deviations from the ORs characteristic of 
solid-state transformation products for this system, which are strongly affected by interfacial strain energy, implying here that matrix and precipitate crystals formed concomitantly from the liquid.

\section{References:}

[1] Kline J.E., Leonard J.P., Applied physics letters 86 (2005), p.201902

[2] McKeown J.T. et al., Acta Materialia 65 (2014), p.56

[3] Gill S.C. et al., Acta Metallurgica Materiala 40(1992), p.2895.

[4] Gill S.C., Kurz W., Acta Metallurgica Materiala 41(1993), p.3563.

[5] Kurz W, Gilgien P., Materials Science and Engineering A 178(1994), p.171.

[6] Work was performed under the auspices of the U.S. Department of Energy, Office of Basic Energy Sciences, Division of Materials Sciences and Engineering for FWP SCW0974 by Lawrence Livermore National Laboratory under Contract DE-AC52-07NA27344. The research activities at the University of Pittsburgh received support from the National Science Foundation, Division of Materials Research, Metals \& Metallic Nanostructures program through Grant No. DMR 1105757.

Figure 1: a) BF TEM images of a representative melt-pool produced by laser irradiation, $b$ ) HAADF STEM image obtained from the melt-pool edge showing the finely separated $\theta^{\prime}$ $\mathrm{Al}_{2} \mathrm{Cu}$ precipitates (bright) within the columnar Al grains (gray) formed during rapid crystal interface movement.
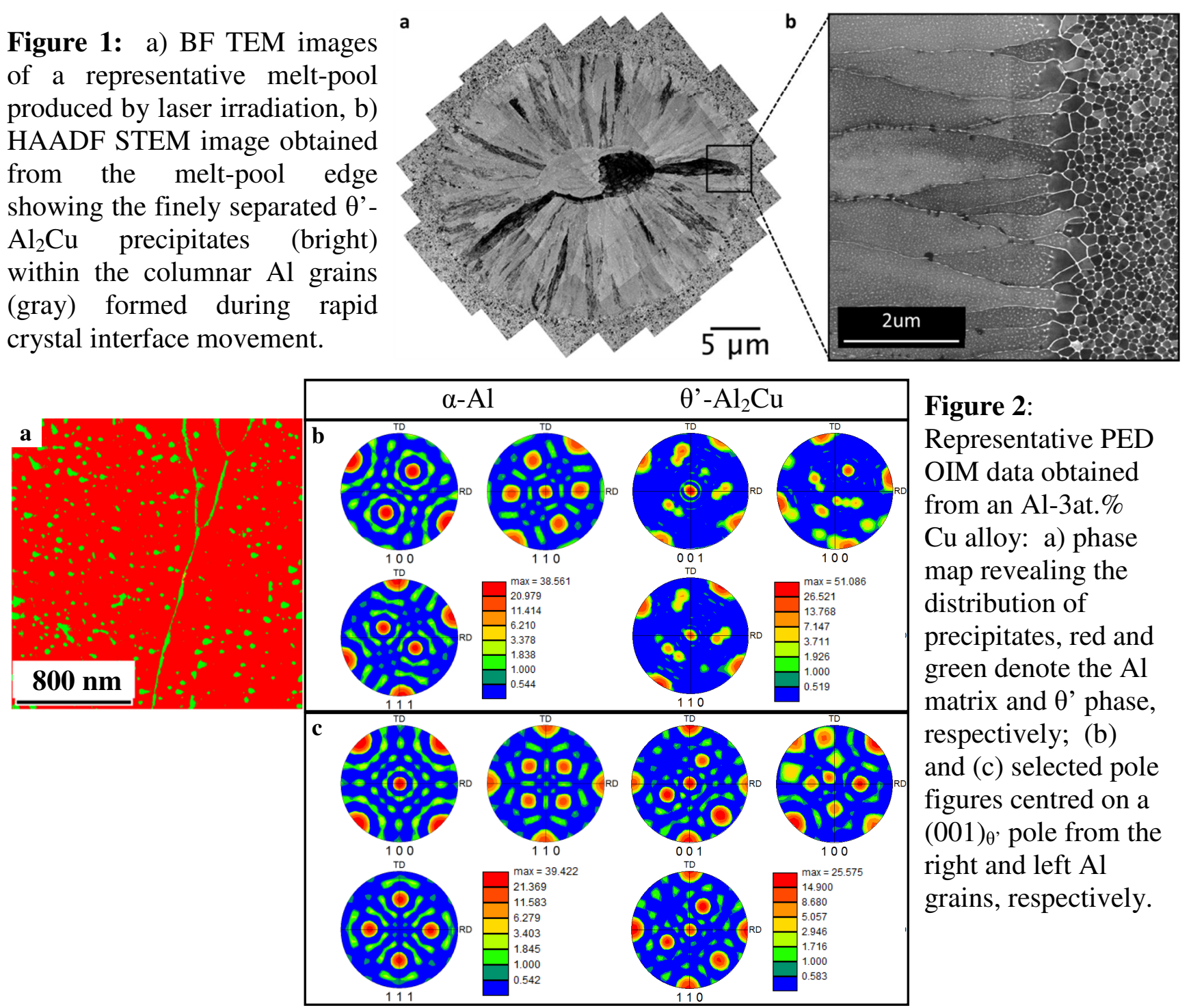

Figure 2:

Representative PED OIM data obtained from an Al-3at.\% $\mathrm{Cu}$ alloy: a) phase map revealing the distribution of precipitates, red and green denote the $\mathrm{Al}$ matrix and $\theta$ ' phase, respectively; (b) and (c) selected pole figures centred on a $(001)_{\theta}$, pole from the right and left $\mathrm{Al}$ grains, respectively. 